Portland State University

PDXScholar

\title{
Analytic Thinking Predicts Vaccine Endorsement: Linking Cognitive Style and Affective Orientation Toward Childhood Vaccination
}

Daniel A. Anderson

Portland State University

Follow this and additional works at: https://pdxscholar.library.pdx.edu/honorstheses

Part of the Public Health Commons

Let us know how access to this document benefits you.

Recommended Citation

Anderson, Daniel A., "Analytic Thinking Predicts Vaccine Endorsement: Linking Cognitive Style and Affective Orientation Toward Childhood Vaccination" (2015). University Honors Theses. Paper 215. https://doi.org/10.15760/honors.220

This Thesis is brought to you for free and open access. It has been accepted for inclusion in University Honors Theses by an authorized administrator of PDXScholar. Please contact us if we can make this document more accessible: pdxscholar@pdx.edu. 
Analytic Thinking Predicts Vaccine Endorsement:

Linking Cognitive Style and Affective Orientation Toward Childhood Vaccination

by

Daniel A. Anderson

An undergraduate honors thesis submitted in partial fulfillment of the

requirements for the degree of

Bachelor of Science

in

University Honors

and

Psychology, Anthropology

Thesis Adviser

Dr. Wayne Wakeland, Ph.D

Portland State University

2016 


\begin{abstract}
Though popular opinion in the US is favorable toward vaccination, a growing hesitancy to vaccinate children threatens rates of uptake and coverage. In response, researchers now study psychological factors thought to influence vaccine-decisions, as having this information might be useful in addressing vaccine hesitancy in the clinic and beyond. The present thesis reviews evidence from this body of work, and shares results of a new study on the influence of analytic and intuitive thinking styles upon endorsement of childhood vaccines. In a national sample $(N=$ 543), analytic thinking predicted endorsement alone and in the presence of covariates in a regression model, while intuitive thinking's relation to endorsement in the model was statistically unclear, and so did not support or refute claims in the literature suggesting this association. Implications and limitations of results, as well as possible directions for future research are discussed in detail.
\end{abstract}

Keywords: vaccines, vaccination, vaccine hesitancy, vaccine endorsement, cognitive style, dual process model, dual processes, analytic thinking, intuitive thinking, need for cognition, faith in intuition 
Analytic Thinking Predicts Vaccine Endorsement:

Linking Cognitive Style and Affective Orientation Toward Childhood Vaccination

\section{Introduction}

Though public opinion toward vaccination is positive in the US, and coverage for most vaccines recommended by the CDC hovers above 90\% (Kahan, 2014; WHO, 2014), hesitancy toward vaccination of children is increasing, as are rates of infection with some vaccinepreventable diseases (Glanz et al., 2013; Gostin, 2015; Omer, Richards, Ward, \& Bednarczyk, 2012; WHO, 2014). In response, researchers now study a multitude of influences upon individual and parental vaccine decision-making, including economic, educational, sociocultural, and psychological factors, suggesting such knowledge is useful in addressing this issue in the clinic and beyond (Boom \& Cunningham, 2014; Dubé et al., 2013; Gupta, 2010; C. M. Poland, Jacobson, Opel, Marcuse, \& Poland, 2014; C. M. Poland \& Poland, 2011; Salmon et al., 2005). In the present thesis, evidence from the psychological branch of this literature is reviewed and results from a new study are shared. The study accessed an online US sample $(N=543)$, testing whether intuitive and analytic thinking styles influence likelihood of endorsement of childhood vaccines/vaccination.

\section{Literature Review and Central Terms}

\section{Hesitancy versus endorsement of childhood vaccination: Definition and explanation}

General vaccine hesitancy/endorsement has been studied by Kahan (2014), who concludes that individual evaluations of childhood vaccine safety are predominantly motivated by emotion. Surveying a large national sample $(N=2,316)$, Kahan found that attitudes toward vaccines in the US are predominantly positive, while identifying an emotional, affective orientation toward vaccines as the primary factor underlying most individual vaccine risk assessments, as opposed 
to calculation of objective risks and benefits of vaccination. These results concur with earlier research on public vaccine knowledge and attitudes in North America, which has found that although most respondents tend to have minimal declarative knowledge on vaccine risk and safety, they still tend to endorse vaccines (Ritvo et al., 2003).

Kahan draws centrally upon Slovic, Peters, Finucane, \& MacGregor's (2005) paper on the role of affect in decision making and risk-judgments. There, it is concluded that in potentially risky scenarios, people tend to judge the options that feel right to them as the safest, often completely failing to calculate objective odds of risk. Reviewing the literature on reasoning and cognitive biases in risk assessments, they conclude that

... [o]ne cannot assume that an intelligent person can understand the meaning of and properly act on even the simplest of numbers, not to mention more esoteric measures or statistics pertaining to risk, unless these numbers are infused with affect. Thus, the forms of [quantitative] information that people take for granted as meaningful, and that they expend immense effort and expense toward gathering and disseminating, may be illusory. (2015: S39).

Central to Kahan's (2104) evidence is a strong inverse correlation between risk and benefit perceptions of childhood vaccines in the sample $(r=-.77, p<.001$, p. 22$)$. Kahan notes ...the best evidence that someone is engaged in ... self-conscious and informed weighing [of risk/benefit] is the independence of her assessments of a putative risk source's risks and benefits. Highly congruent [i.e., correlated] perceptions of costs and benefits, in contrast, imply a gestalt form of judgment driven by an affective appraisal (2014: 23).

That is, since there is so much variation in how safe/dangerous vaccines are, person-to-person 
and situation-to-situation ${ }^{1}$, those who weighed vaccine decisions largely on the data would probably show a lower correlation between risk and benefit perceptions than those who held a unidimensional attitude. Thus, since the Pearson coefficient there is strong, it is inferred that the majority of the population more often makes affective, rather than intellect-driven vaccine decisions.

This is a compelling possibility, and recommends continued research. The present study attempted to replicate and move forward with this finding of association between risk and benefit perceptions, asking how much, if at all, intellectual rather than affective mental activity might actually influence childhood vaccine endorsement, and how (again, if at all) might more intuitive people vary in their deployment of affect versus intellect in their vaccine decisions? Intellectual versus affective aspects of cognition and personality are explored in the next section.

\section{Dual process models and cognitive style}

Most researchers in psychology agree that brains handle the sensory and cognitive information of daily life using two discrete information-processing systems. One system is analytic, rational, and intentional, the other intuitive, automatic, and affective; (Evans \& Stanovich, 2013; Evans, 2008, 2012; Kahneman, 2011; Tversky \& Kahneman, 1974). Each of these systems go by multiple names in the literature (see Evans \& Stanovich, 2013 for a review), and are referred to here as mainly as the analytic and intuitive systems, following Epstein (1994, 1998, 2014). The analytic system is associated with intentional work on effort-demanding tasks, like calculation and memorization of terminology, while the intuitive system is basically effortless and instinctive: it is defined by perceptions, emotions, and 'going by the gut'. Most moment-to-moment thinking is the work of the intuitive system, but this automaticity can be

\footnotetext{
${ }^{1}$ See Jacobson et al. (2001) for a discussion of objective risks associated with vaccines.
} 
'intervened' upon by the analytical system to focus on harder tasks (Evans, 2008).

Conceptualization of human thinking in this dichotomous fashion goes back at least to William James's discussion on the regulation of 'passions' (1890), and today is commonly called dualprocess theory (Evans \& Stanovich, 2013; Evans, 2008).

Evidence suggests different people employ and rely on each of these systems to varying degrees - some people predictably favor use of the intuitive system, others tend to use the rational system more (Chaiken \& Trope, 1999; Epstein, Pacini, Denes-Raj, \& Heier, 1996; Epstein, 1994; Evans \& Stanovich, 2013; Evans, 2008; Pacini \& Epstein, 1999). In personality psychology, specifically in Cognitive Experiential Theory, or CET (Epstein, 2014), the terms intuitive cognitive style and analytic cognitive style refer to individual persons' overall orientation in this dichotomy - this is 'how intuitive/analytical they are' in the day-to-day thinking. In CET, this orientation is commonly measured in the person with a one of several versions of a psychometric called the Rational/Experiential Inventory, or REI (Epstein et al., 1996; Pacini \& Epstein, 1999; see Materials section). Though CET conceptualizes interactions between the two systems as ongoing throughout day-to-day thought, the analytic and intuitive factors of the REI generally do not correlate - thus the two systems can be considered separate, though interactive (Epstein et al., 1996; Pacini \& Epstein, 1999).

Through such interactions, the two systems can 'train' each other: activities that take effort in the beginning become automatic as expertise is gained (Klein, 1999; Sladek, Bond, \& Phillips, 2010), just as education in analytically vigorous disciplines, like the natural sciences, seems to lead to less inclination toward behaviors and beliefs associated with the intuitive system $^{2}$ (Aarnio \& Lindeman, 2005). Individuals differ in their level of partaking in activities

\footnotetext{
${ }^{2}$ Beliefs associated with higher use of the intuitive system are discussed in coming sections.
} 
that nurture and train the two systems to varying degrees (for multitudes of reasons), and so differences in cognitive style between individuals can be described as mediated by individual's unique circumstances, including and especially environmental/cultural influences (see Buchtel \& Norenzayan, 2009).

Heuristics. Crucially, intuitive people tend more often than analytic people to use heuristics and cognitive biases, or 'quick and dirty' mental shortcuts in their decision-making, such as judging the likelihood of something based on how easily it springs to mind, as opposed to considering objective probabilities (Stanovich \& West, 1998; West, Toplak, \& Stanovich, 2008; see Tversky \& Kahneman, 1973). Indeed, use of heuristics is considered a hallmark of intuitive thinking, while use of effortful, systematic reasoning is considered the hallmark of the analytic system $^{3}$ (see Epstein, 1994; Evans, 2008; Gilovich, Griffin, \& Kahneman, 2002; Kahneman, 2011; Tversky \& Kahneman, 1983). What makes this point crucial is that Kahan's (2014) conceptualization of vaccine endorsement is heuristic: it is affective rather than intellectual. This thesis asks: might then the outcomes of Kahan's intuitively-rooted measurement (that is, vaccine hesitancy/endorsement) be influenced by the general rate at which a person employs heuristics in everyday thought - i.e., their general reliance on the intuitive system? And what about an overall tendency toward use of the analytic system? Literature reviewed below suggests both these associations might exist.

\footnotetext{
${ }^{3}$ It must be noted that heuristics are evolutionarily adaptive: just because they often lead to objectively inaccurate conclusions does not negate the fact that are the product of natural selection, and so for millennia brought primates to accurate enough conclusions to ensure the fecundity of hominids (Cosmides \& Tooby, 1996).
} 
Use of heuristics by the vaccine hesitant. Variation between individuals in their tendency toward use of analysis versus heuristics has been argued to influence their vaccine decisions: it is suggested in a growing literature that heuristic decision-making might underlie a significant amount of vaccine hesitancy (Gupta, 2010; Jacobson, Targonski, \& Poland, 2007; Poland et al., 2014; Poland \& Poland, 2011; Poland, Jacobson, \& Ovsyannikova, 2009). Indeed, it has been suggested that medical professionals should adapt patient/parental-counseling on vaccine safety to the fact that people might reason heuristically, rather than simply presenting patients/parents with data on vaccine safety and expecting them to act as rational agents - which is purportedly common practice in clinical settings (Gupta, 2010; Poland \& Poland, 2011). In light of this suggestion, Poland and others (2014) offer a detailed list of heuristics and biases known to cognitive scientists, and which might influence the decision making of vaccine-hesitant individuals and parents. Several are reviewed below, with both quoted and paraphrased explanations, along with the citation of the original research on the specific heuristic/bias:

(1) The confirmation bias, or the tendency to seek confirmatory evidence while discounting contradictory evidence (see Nickerson, 1998 in Poland, et al., 2014) seems at work, for instance, in individuals' acceptance “... as evidence of cause and effect reports of a child being diagnosed with autism in near proximity to receipt of [the] MMR vaccine” (p. 346) thus confirming for them this widespread belief (see Poland \& Jacobson, 2012), even after being presented with scientific evidence by medical researchers that contradicts this belief's soundness.

(2) The representativeness heuristic, or judging the likelihood of an event considering its superficial resemblance to other events (see Tversky \& Kahneman, 1974 in Poland, et. al, 2014) might underlie individuals' associating maladies and vaccination. A person might 
consider such a link valid to the extent that such situations resemble rumored and/or real-life incidents of vaccine contamination and/or adverse effects of vaccines on some individuals (see also Jacobson et al., 2001 for exploration of documented adverse effects of vaccines). (3) The omission/commission bias, or the tendency to perceive possible adverse effects of inaction as preferable to possible adverse effects of action, irrespective of the objective risks of each option, might be at work in the person's perception of disease contraction as preferable to a presumed side effect of a vaccine (see Asch et al., 1994 in Poland et al., 2014; Meszaros et al., 1996).

(4);(5) Belief perseverance, or the tendency to hold beliefs even after being confronted with contradictory data (see Nestler, 2010 in Poland, et al., 2014), as well as risk compression, or a tendency to overestimate the prevalence of statistically rare risks (see Fischhoff, 1993 in Poland, et. al, 2014) are each tautologically present in many persons' decision to reject vaccines.

(6) Attributional frameworks, or the construction of compelling causal explanations for events (which are highly memorable) - despite incongruences in the inference of causality given what data actually show (see Nestler, 2010 in Poland, et al., 2014) - seems present in the emotionally compelling personal memories people often reference as causal to a decision to not vaccinate.

(7) Avoidance of ambiguity, or the tendency to consider a known risk as less risky than an ambiguous one (see Baron, 2000 in Poland, et. al, 2014) is purportedly seen in a person's perception of infection with diseases as being less risky than whatever possible side effects of vaccines. 
Poland \& Poland (2011) also offer a related taxonomy of common patterns in reasoning they associate with lower vaccine endorsement, which includes $(a)$ "denialist" thinking styles associated with disregard of scientific fact and acceptance of the information presented in conspiracy theories; $(b)$ fear-based thinking styles motivated by subjective feelings of fear regarding vaccines; (c) "right-brained" (emotional) thinking styles which fail to grasp what statistical figures reflecting vaccine risks actually mean, and $(d)$ "heuristic" thinking, or the reliance on mental shortcuts like those already outlined above. With regard to the present thesis, note that these thinking styles indicate reliance on intuition: fear-response-instincts, 'right brained-ness' (emotional thinking style), and of course heuristics are all prototypically intuitive mental features (Epstein, 1994; Evans, 2008; Pacini \& Epstein, 1999). As for the "denialist" reasoning (i.e., reasoning focused on conspiracy theories), this will be approached in a coming section.

Finally, Jacobson et al. (2007) also offer a taxonomy of reasoning flaws observed among those who reject vaccines, which they derive from Gilovich's (1991) critique of reasoning flaws in modern society. There, vaccine hesitant individuals are noted as showing high rates of seeking and "...find[ing] order and predictability in random data" (Jacobson et al., 2007: 3147) where they expect it (for instance, seeing danger in vaccination where it does not exist according to the data), and "difficulty in detecting and correcting biases in incomplete and unrepresentative data... [along with] ...eagerness to interpret ambiguous and inconsistent data to fit theories and expectations" (p. 3147). The thinking habits noted in Jacobson, et al. seem, like the preceding examples, rooted in a favoring-of and/or failure-to-work-against the intuitive system when making decisions under uncertainty/the reliance on heuristics rather than data. 
These papers seem to suggest lower (i.e. negative) orientation toward vaccines, or hesitancy rather than endorsement, might be observed among more intuitive individuals, as they are the ones (theoretically) more prone to use heuristics, and use of heuristics is associated with hesitancy. However, these papers only imply that higher levels of analytic thinking might be associated with vaccines endorsement.

\section{Minimal research on analytic thinking and vaccine endorsement.}

Indeed, only two papers cited above note analytic cognition's possible role in the decision to vaccinate, touching on this system's association with data-focus (as opposed to emotionfocus) in the evaluation of claims and decision-making (Gupta, 2010; Poland \& Poland, 2011).

Overall, the papers cited above say little about the analytic mind at all, and mostly discuss heuristics and biases in reasoning. Perhaps the authors choose to focus on the inverse association of vaccine endorsement with the intuitive system because lower rates of endorsement are of more pressing interest than factors associated with higher endorsement of vaccines. It also is possible that these authors conceptualize dual process models as opposite ends of a single scale, rather than as separate systems (see Evans \& Stanovich, 2013 for critisisms of this concpetualization), and so assume that since heuristics are associated with vaccine hesitancy, it simply goes without saying that analytic thinking would associate with endorsement.

Although this seems a simple exercise in equating 'like with like', the literature supports this conjecture at least at the level of predictor and outcome-type: an analytic cognitive style has been positively associated not just with higher education level, but with an overall higher level of acceptance of scientifically founded beliefs, and concurrent lower levels of belief in pseudoscience; supernatural phenomena; the paranormal, and belief in conspiracy theories (Aarnio \& Lindeman, 2005; Browne, Pennycook, Goodwin, \& McHenry, 2014; Genovese, 2005; 
Gervais \& Norenzayan, 2012; Gervais, 2015; Pennycook, Cheyne, Seli, Koehler, \& Fugelsang, 2012; Swami, Voracek, Stieger, Tran, \& Furnham, 2014). For instance, greater endorsement of evolution, a foundational scientific principle, along with rejection of creationism, was recently found by Gervais (2015) to be predicted by higher levels of analytical thinking, while Gervais and Norenzayan (2012), along with (Browne et al., 2014) observed higher scores on measures of analytical thinking associated with lower religiosity and spirituality. Further, Aarnio and Lindeman (2005) found that analytic thinking mediated a negative relationships between education level and belief in the paranormal among Finnish university students, while increases in analytical thinking were found associated with lowered propensity to believe conspiracy theories in Swami et al. (2014) - which presumably equates to a greater acceptance of more mainline, scientifically founded beliefs (see Kata, 2012 - more on conspiracy theories will follow).

These results support the notion that higher levels of analytical thinking underlie higher likelihood of holding scientifically rooted beliefs, while lower analytic thinking is associated with higher likelihood of holding superstitious beliefs; beliefs in magic and the supernatural, and other beliefs, which “...have no epistemic warrant” according to science (Lobato, Mendoza, Sims, \& Chin, 2014: abstract; see Subbotsky, 2014). It would seem logical, given these findings, that other beliefs distanced from scientific consensus, e.g. beliefs underlying a hesitancy to accept medical consensus on vaccine safety (discussion of such beliefs follows shortly), might inversely correlate with measures of analytic thinking style.

\section{The need for more direct, empirical studies of association}

To return to the link between heuristics and vaccine hesitancy noted in the literature, a methodological issue must be noted: in all of the papers reviewed, this relationship is only 
suggested, and is not empirically measured. While these papers are by respected scholars, they tend to rely on informed conjecture rather than quantitative support. Further (and discouragingly for this study), the one paper located during the review that did conduct a direct test of association, using psychometrics for these variables, found no association at all (Browne, Thomson, Rockloff, \& Pennycook, 2015). Certainly, this was unexpected. Given the papers reviewed above and their claims to having identified heuristic processing as highly present in the vaccine hesitant compared to endorsers - along with the implication that analytic thinking is related to endorsement - it would seem that detection of such an association would be likely.

That said, Browne et al.'s methods could be criticized, as their operationalization of a dual-process model is perhaps too limited. The psychometric they use (Frederick, 2005) specifically measures intervention upon the intuitive system by the analytic system, via exposing subjects to mathematical story problems where a supposedly intuitive, heuristic answer is apt to spring to mind (which is contrary to the mathematical answer). The idea is that analytic thinkers tend to get it right, and intuitive thinkers tend to get it wrong. Now, while capacity toward analytic intervention upon intuition would logically give some indication of an individual's general cognitive style, this criterion is perhaps too limited to truly test the person's overarching cognitive style. Indeed, deployment of rational and intuitive cognitive systems in general covers a much larger realm of human life than understandings of numerical proportions and quantitative rates of change (this is the mathematical criteria in Frederick, 2005): these systems are deployed in memory, perception, attribution, and multitudes of other psychological domains; perhaps all of them (Epstein, 2014; Kahneman, 2011).

In light of this criticism, and in light of the lack of conclusive, quantitative/empirical research on the relationship of heuristics and cognitive style to vaccine endorsement, a new study 
was done, and is described in the remainder of this thesis (after a brief discussion on the nature of some beliefs underlying vaccine hesitancy is necessary).

\section{A brief caveat on conspiracy theories.}

While designing the study, colleagues noted that belief in conspiracy theories underlies much vaccine hesitancy. A review of the literature suggested that, indeed, both vaccine endorsement and cognitive style are associated with belief in conspiracy theories: belief in specific conspiracy theories is thought to compel individuals toward vaccine hesitancy (CDC, 2006; Coady, 2006; Diethelm \& McKee, 2009; Feldman-Savelsberg, Ndonko, \& Schmidt-Ehry, 2000; Jolley \& Douglas, 2014; McConnachie \& Tudge, 2013; Wilson, Larson, Chiu, \& Schulz, 2015), while specific heuristics and reasoning errors (e.g., the representativeness heuristic and confirmation bias reviewed in the earlier section) have been associated with acceptance of conspiracy theories and the assumptions which underlie them (Brotherton, French, \& Pickering, 2013; Brotherton \& French, 2014; Leman \& Cinnirella, 2007). Further, the heuristic role of fear and emotion in the acceptance of conspiracy theories is well-explored (Darwin, Neave, \& Holmes, 2011; Jolley \& Douglas, 2014; Leman, 2007). Regarding analytic thinking, experimentally induced increases in analytical thinking among test subjects have been associated with lowered levels of acceptance of conspiracy theories (Swami et al., 2014). Given this truncated review (far more research on conspiracy theories was reviewed, and is omitted here for brevity), it was determined that a metric of belief in conspiracy theories should to be included in the study as a covariate.

\section{A final caveat: Demographics/politics.}

Though not of central interest here, age, education, sex, and parental status, as well as political orientation are all known to associate with vaccine endorsement/hesitancy in the 
individual and parent (see Boom \& Cunningham, 2014; Dubé et al., 2013 for reviews), and so measures for these parameters were also included in the study as covariates. Where possible, they were measured so as to be comparable to 2014 Census data.

\section{Study Rationale and Hypotheses}

\section{Study rationale}

Theoretical links between cognitive style and vaccine endorsement found during the literature review suggested a direct association between cognitive style and vaccine endorsement might be detected in a national survey holding other relevant factors constant, and that this relationship might be bi-directional, with intuitive cognitive style associating negatively, and analytic cognitive style associating positively with vaccine endorsement.

\section{Hypotheses}

In a national sample, analytic cognitive style will positively associate with vaccine endorsement, while intuitive cognitive style will negatively associate with vaccine endorsement. These relationships will be observed holding constant political and demographic covariates, along with beliefs in conspiracy theories.

\section{Methods}

\section{Ethics Statement}

The Institutional Review Board at Portland State University approved the study and all respondents gave informed consent.

\section{Participants and procedure}

After aggregating questionnaires (see Materials section below) into a digital, online format using Qualtrics online software (Qualtrics, Provo, UT), a national US sample was accessed $(N=$ 603, age 18+) and the survey administered via Mechanical Turk (Amazon.com, Inc. Seattle, 
WA). Mechanical Turk, or Mturk, is a paid online service where researchers can recruit and administer surveys to participants, as well as deliver compensation. Mechanical Turk's participant pool has been validated in multiple papers as more representative of the US population than traditional participant pools accessed in mail and telephone surveys; comparable in response patterns to traditional pools and, finally, less expensive than other commercial online participant recruitment tools like surveymonkey.com (see Brandon, Long, Loraas, MuellerPhillips, \& Vansant, 2013 for a review).

Piloting the survey, average completion time was 12 minutes 43 seconds. Cutoff for inclusion was set generously at 5 minutes, which excluded some respondents $(n=40)$. A few more were excluded for failing to provide completion codes on Mturk $(n=18)$, while two $(n=$ 2) had to be excluded for incomplete surveys. This left $N=543$ out of the original $N=603$ in the final dataset. All results were similar with or without these exclusions.

A note on demographic variables. Data were weighted so that the variable sex matched the 2014 census (females $=50.8 \%$, males $=49.2 \%$ ) because females were overrepresented in the sample by nearly $n=100$, with males $n=223$, females $n=320$. Single variable weighting was performed by dividing the population-percentage of each sex by its percentage in the sample, and then multiplying each observation by the coefficient of its respondent's sex. This resulted in males $n=267$, females $n=276$.

\section{Materials}

Though all measurements were taken at the ordinal level, alpha values $(\alpha)$ were calculated to offer a suggestion of reliability per instrument. Future analysis could explore the use of alternative reliability assessments designed for use on ordinal data (see Gadermann, Guhn, \& Zumbo, 2012). 


\section{Central measures: Vaccine endorsement and cognitive style}

Vaccine endorsement. Kahan's (2014) measure of affective orientation toward childhood vaccines $(n=21)$ was used to gauge level vaccine endorsement. This latent parameter is argued by its author to underlie individual risk/benefit perceptions of vaccines, level of support for universal immunization, and level of trust in the judgment of health officials/professionals. Crucially, this instrument is not specially tailored for use on parent samples, as were many measures of orientation toward vaccine encountered during the review. It therefore was deemed most appropriate for analysis of general endorsement of childhood vaccines in the adult population. Nineteen items from Kahan's instrument were utilized, and two were excluded because they were related to perception of vaccine acceptance within the population rather than one's personal orientation toward vaccines. This represented a single measure called vaccine endorsement here, which used 6-point and 8-point likert scales $(\alpha=.959)$. Example items include "I would have a negative view of parents who decided not to have their child receive generally recommended childhood vaccinations", and "children who receive generally recommended childhood vaccinations have a higher risk of developing autism than children who are not vaccinated".

Cognitive Style. Cognitive style was measured with a 10-item version of the Rational Experiential Inventory (REI-10) (Epstein et al., 1996), a two factor instrument gauging 'faith in intuition' ( $n=5 ; \alpha=.907)$ and 'need for cognition' $(n=5 ; \alpha=.853)$, factors that purportedly gauge individuals' overall engagement with dual processes of cognition in day-to-day thinking. Following Epstein (1994, 1996, 2014), here the overall consilience of dual-process models is taken to allow 'need for cognition' to stand in for 'analytic cognitive style', while 'faith in intuition' stands in for 'intuitive cognitive style'. 
This instrument is a simplified version of its 40-item predecessor (REI-40), which is used in research noted throughout the literature review to measure cognitive style. It uses a 5-point Likert scale, asking respondents to rate their belief in the truth or falsity of statements such as "I prefer to do something that challenges my thinking abilities rather than something that requires little thought" ('need for cognition'), and "I trust my initial feelings about people" ('faith in intuition') between 1 (completely false) and 5 (completely true).

\section{Covariates: Conspiracist beliefs and political orientation}

Conspiracist belief. Brotherton, et al.'s (2013)'s 15-item Generic Conspiracist Beliefs Scale measures a cluster of assumptions people might make about how typical conspiracist activity is in the world: assumptions of global governmental malfeasance, extraterrestrial coverups, personal wellbeing conspiracies (e.g., fluoride in drinking water is poisonous/vaccines are secretly threats to the personal wellbeing of the population), and control of information by powerful forces like governments and secret societies. These assumptions are taken to underlie acceptance of hosts of specific conspiracies in the individual (e.g., 9/11 attacks were part of secret governmental agendas; the Holocaust did not happen).

It is a propensity to hold such beliefs that is of interest here, rather than whatever specific conspiracies might be held by individuals, as it is this general factor that shows association with cognitive style, as well as the overall mistrust of the power structures of mainstream culture that arguably underlies much vaccine hesitancy (see Kata, 2010, 2012). The authors sum the conspiracist belief measure's items into a single scale, as was done in the present study $(\alpha=$ .936). This instrument uses a 5-point Likert scale, and presents items such as "the power held by heads of state is second to that of small unknown groups who really control world politics", and "groups of scientists manipulate, fabricate, or suppress evidence in order to deceive the public". 
Political orientation. A single item measure of political orientation was included as well. This five-point measurement ranges from " $1=$ very liberal" to " $5=$ very conservative". Despite being minimal in structure, it has shown high predictive/construct validity in experiments, predicting specific voting patterns with remarkable accuracy (Jost, 2006) and revealing neural correlates of political orientation (Kanai, Feilden, Firth, \& Rees, 2011). While not of central interest here, recent research drawing on US government surveys suggests a significant link exists between conservative political identification and lower vaccine orientation (Lupton \& Hare, 2015), so this variable was included here as a relevant covariate.

\section{Results}

Figures were generated and data cleaning performed using OS Numbers (Apple, Inc. Cupertino, CA) and IBM SPSS version 21.0 for Macintosh (IBM, Armonk, NY). Statistical analyses were also performed using SPSS. All data will be made available on the author's website.

\section{Descriptive statistics}

After weighting the data, frequencies for demographic variables (sex, age range, parental status, level of education) were calculated and are shown in Tables 1-3 in Appendix A. Comparing level of education and age range to 2014 census data with chi-square goodness of fit tests, it was found that proportions per level of education in the sample were highly congruent with those in the 18 and over US population $\left(\chi^{2}(14)=439.3091, p<.001\right)$, while proportions per age range were not $\left(\chi^{2}(11)=4.298, p=.960\right)$, with younger individuals being overrepresented in the sample and the elderly barely being represented at all (see Table 2). This limitation was accepted and the analyses moved forward, as the sample showed higher diversity in this variable than might have been observed, for example, in most undergraduate samples. 
Preliminary analyses showed vaccine endorsement was highly positively skewed (see Figure 1), indicating a high rate of endorsement in the sample that concurs with earlier research, $\operatorname{Min}=22, \operatorname{Max}=122, M d n=100, Q 1=79, Q 3=112$. 'Need for cognition', $\operatorname{Min}=5, \operatorname{Max}=25, M d n=19, Q 1=15, Q 3=19$, and 'faith in intuition', Min $=5, M a x=25, M d n=19, Q 1=16, Q 3=19$, showed somewhat less pronounced, but still long, left tails. Conspiracist belief was more normal, $\operatorname{Min}=16, \operatorname{Max}=80, \operatorname{Mdn}=43, Q 1$ $=32, Q 3=52$, while political orientation was skewed toward lower (more liberal) scores, $\operatorname{Min}=1, \operatorname{Max}=5, M d n=3, Q 1=2, Q 3=3$. These overall non-normal distributions, along with the ordinal level of measurement suggested using nonparametric tests to examine association between variables.

Chi square tests failed to detect statistically significant differences in scores of vaccine endorsement between sexes, $\chi^{2}(82)=92.581, p=.199$, or between parental statuses, $\chi^{2}(82)=96.488, p=.131$, however these categorical variables were associated with multiple study variables (see Tables 4-5 for results of tests of independence between parental-status/sex and study variables), and so they were included in the regression models discussed shortly.

\section{Validation of the affective orientation construct}

After calculating descriptive statistics, positively and negatively valenced items

from the vaccine endorsement instrument were summed separately into two variables and plotted in a scatterplot (see figure 2). Visual analysis confirms Kahan's finding of a strong, negative association between risk and benefit assessments of vaccination, and suggests, by Kahan's standards at least, a predominantly affective/heuristic approach to vaccine orientation throughout the population rather than a high amount of calculation of 
risk/benefits. The scatterplot also concurs with the high level of vaccine endorsement in the population seen in Figure 1, with a large clustering of scores showing an overall positive opinion of childhood vaccines. A very strong and highly significant Pearson correlation coefficient confirmed the association, $r=.872, p<.001$. This finding will be returned to after testing the central hypotheses.

\section{Nonparametric correlations}

Spearman rank correlation coefficients were calculated between study variables and are shown in Table 6. Concurring with previous research on the REI, 'need for cognition' and 'faith in intuition' were not associated, $r_{s}=-.001, p=.975$. In support of fundamental assumptions of dual process models, 'need for cognition' positively associated with level of education, $r_{s}=.109$, $p=.011$, while 'faith in intuition' showed a negative association there $r_{s}=-.200, p<.001$.

Offering mixed support for research reviewed, conspiracist belief showed a positive association with 'faith in intuition' $r_{s}=.205, p<.001$, but no association with 'need for cognition', $r_{s}=-$ $.080, p=.064$

In support of the hypothesis, 'need for cognition' showed a weak but significant association with vaccine endorsement at $r_{s}=.154, p<.001$, while, contrary to the hypothesis, 'faith in intuition' showed no association there, $r_{s}=-.026, p=.552$. Vaccine endorsement showed a moderate, negative associations with conspiracist belief, $r_{s}=-.486, p<.001$, and a weak positive association with level of education, $r_{s}=.110, p<.001$. Political orientation and vaccine endorsement were negatively associated, $r_{s}=-.307, p<.001$, indicating lower levels of vaccine endorsement among more conservative respondents, and higher scores on vaccine endorsement among the more liberal. This is in line with recent research noted in the Measures section. Liberalness was associated with higher 'need for cognition', and conservativeness with 
cognition', $r_{s}-.132, p=.001$, while 'faith in intuition' did not associate with political orientation $r_{s}=.079, p=.065$.

Aside from these notable associations, an overall high level of shared association among variables was observed (see Table 6). For example, education level showed weak but significant associations with nearly all variables. This suggested entering all variables into an explanatory model to suggest at what degree cognitive style contributes to vaccine endorsement holding all other variables constant.

\section{Ordinal regression analyses}

Recoding and tests of assumptions. The variables vaccine endorsement, 'need for cognition', and 'faith in intuition' were recoded into high, medium, and low score groups at their $33^{\text {rd }}$ percentiles. This was done both to ease presentation of results shown in figures and because results from all models using the raw data failed to meet the assumption of proportional odds required to perform ordinal regression (with $p$ 's $<.05$ in testing a null hypothesis of proportional odds). That is, ordinal regression assumes the ability to predict values of the dependent variable with equal odds at any level of the independent variable. This assumption was met after recoding the variables into the three-tier groups, $p$ 's $>.05$. This result held across all models tested. Tests on the final model also showed that despite shared associations throughout the data, multicollinearity was not an issue (Tolerances, .816 to .976 , VIFs, 1.228 to 2.286).

Ordinal regression models. In the first model (all models are shown in Table 7), 'need for cognition' was tested singularly. It significantly predicted higher odds of vaccine endorsement at an odds ratio of $1.473(95 \% \mathrm{CI}, 1.218$ to 1.782$)$, Wald $\chi^{2}(1)=15.984, p<.001$, (see Figure 3 in Appendix B). Adding 'faith in intuition' to the model with 'need for cognition' (model 2, Table 7), 'need for cognition' continued to associate similarly at an odds ratio of 1.475 
(95\% CI, 1.220 to 1.784$)$, Wald $\chi^{2}(1)=16.076, p<.001$, while 'faith in intuition' failed to associate with higher or lower odds of vaccine endorsement, at an odds ratio of 0.954 (95\% CI, 0.795 to 1.146$)$, Wald $\chi^{2}(1)=0.250, p=.617$. Likewise, due to its showing no correlation with vaccine endorsement, 'faith in intuition' was not entered into a model as a single predictor.

Entering conspiracist belief into the model (model 3), it was found to be a significant predictor of lower odds of vaccine endorsement at an odds ratio of 0.931 (95\% CI, .919 to .944), Wald $\chi^{2}(1)=105.886, p<.001$, while 'need for cognition' continued to significantly and positively predict vaccine endorsement at an odds ratio of 1.401 (95\% CI, 1.148 to 1.709$)$, Wald $\chi^{2}(1)=11.010, p=.001$. Contrary to all expectations, this model showed 'faith in intuition' associating positively with vaccine endorsement: higher 'faith in intuition' scores were associated with higher odds of increased scores on vaccine endorsement at an odds ratio of 1.226 (95\% CI, 1.007 to 1.492 ), Wald $\chi^{2}(1)=4.123, p=.042$, once conspiracist belief was entered into the model. These results suggest that, controlling for conspiracist beliefs, 'faith in intuition' and 'need for cognition' are each independent, positive, and significant predictors of vaccine endorsement.

Model 4 included only demographics and political orientation as predictors. There, political orientation, and education emerged as significant predictors of vaccine endorsement, with lower (more liberal) political orientation predicting higher vaccine endorsement, at an odds ratio of $.625(95 \% \mathrm{CI}, .540$ to .724$)$, Wald $\chi^{2}(1)=39.129, p<.001$, and higher level of education predicting higher vaccine endorsement at an odds ratio of 1.096 (95\% CI, 1.008 to 1.912 ), Wald $\chi^{2}(1)=4.573, p=.032$. Adding cognitive style variables to the model (model 5), higher scores on 'need for cognition' predicted higher scores on vaccine endorsement as hypothesized, at an odds ratio of $1.365(95 \% \mathrm{CI}, 1.124$ to 1.658$)$, Wald $\chi^{2}(1)=9.837, p=.002$. Lower (more liberal) 
scores on political orientation again predicted higher scores on vaccine orientation, at an odds ratio of $.637(95 \% \mathrm{CI}, .549$ to .739$)$, Wald $\chi^{2}(1)=35.479, p<.001$. Following results of the earlier model, 'faith in intuition' did not emerge as a predictor, 1.070 (95\% CI, .879 to 1.301$)$, Wald $\chi^{2}(1)=.453, p=.501$, and neither did level of education, 1.09 (95\% CI, 1.000 to 1.187 ), Wald $\chi^{2}(1)=3.844, p=.050$, though it was at the threshold of significance at $95 \%$ confidence. Adding it to the final model (model 6), conspiracist belief emerged as a significant, negative predictor of vaccine endorsement at an odds ratio of .928 (95\% CI, 914 to .941$)$, Wald $\chi^{2}(1)=104.260, p<.001$. As was the case in the earlier models, 'faith in intuition' emerged as a significant, positive predictor of vaccine endorsement upon the inclusion of conspiracist beliefs into the model, at an odds ratio of $1.331(95 \% \mathrm{CI}, 1.076$ to 1.647$)$, Wald $\chi^{2}(1)=6.964, p=.008$, while 'need for cognition' was also a significant, positive predictor of vaccine endorsement in this model, at an odds ratio of $1.336(95 \% \mathrm{CI}, 1.089$ to 1.634$)$, Wald $\chi^{2}(1)=7.711, p=.005$. Political orientation continued to negatively predict vaccine endorsement, as was the case in all models, with lower, more liberal scores predicting higher vaccine endorsement and higher, more conservative scores predicting lower vaccine orientation, at an odds ratio of .610 (95\% CI, .522 to .713$)$, Wald $\chi^{2}(1)=38.336, p<.001$.

Additional analyses. Finally, the correlational analyses between positively and negatively valenced items done both in Kahan (2014) and in the present thesis were repeated for each of the 'need for cognition' and 'faith in intuition' score subgroups (low, medium, high).

Though not central to the hypotheses and so not in the main body of the forthcoming discussion, results from these analyses are essential in clarifying this paper's conclusions, and so are returned to in the final section. 
Associations between positively and negatively valenced items were strong ${ }^{4}(r<-.50)$, for each sub-group. In 'need for cognition' score subgroups, coefficients increased sequentially per score subgroup at relatively small levels of effect (low: $r=-.759, p<.001$; medium: $r=-$ $.859, p<.001$; high: $r=-.931, p<.001$ ). Among 'faith in intuition' score subgroups, the medium and high score subgroups showed similar coefficients (medium: $r=-.838, p<.001$; high: $r=$ $.857, p<.001$ ), while the low score subgroup showed a stronger relationship, $r=-.919, p<001$. This variation is minimal, as no sub-group departed from the pattern within score subgroups of strong Pearson $r$ coefficients.

\section{Discussion}

\section{Summary}

The hypotheses received partial support. Holding covariates constant in several regression models, 'need for cognition' did positively predict vaccine endorsement in a national sample, while intuitive thinking, unexpectedly, also positively predicted vaccine endorsement in several regression models, but only upon inclusion of a conspiracist belief measure.

\section{Unexpected, inconclusive results}

A negative association between intuitive cognitive style and vaccine endorsement had been hypothesized based on a suggestion in the literature that heuristics underlie vaccine-hesitant decisions. Why might the data have deviated so completely from this expectation? Two possibilities are suggested here. One considers a possible error in selection of measurements: Kahan's (2014) instrument is, again, conceptualized as measuring level of affective, rather than intellectual orientation toward vaccines. What the author of the present thesis did not consider is that a general tendency to rely on intuitive judgments, i.e. 'faith in intuition' (which is considered

\footnotetext{
${ }^{4}$ See Rubin, 2012 for classification of weak, moderate, and strong Pearson $r$ coefficients.
} 
highly congruent with affect in dual process models [Epstein, 1994]), might positively associate in the direction of data-skew with any measure of affective orientation once other co-associated predictor variables (here, conspiracist beliefs) are held constant. This no doubt speculative, yet colleagues more experienced in ordinal regression analysis suggested this is plausible, and that state of the art statistical tests should examine this possibility in future work.

It is also possible that, simply, most Americans' intuitive reaction toward vaccines is trust, and so higher use of intuition in day-to-day life is associated with higher vaccine endorsement (but, this association has is not visible without controlling for specific confounds). This would certainly follow Kahan's (2014) and Ritvo et al.'s (2003) conclusions (discussed earlier), and would concur with the general skew toward endorsement in the data.

For clarification, this positive skew indicates that the general public's intuitions concur with the highly complex biomedical and epidemiological concepts underlying both the biological workings-of, and rationale-behind, vaccine-recommendations of doctors and public health officials. Since most people do not have the time to learn the ins-and-outs of these complex concepts, they therefore might actually hold an irrational 'faith in science' (see Farias, Newheiser, Kahane, \& de Toledo, 2013) independent of whatever analytical understanding of these scientific/medical concepts. This would concur with Slovic et al. (2005), that ...[u]sing an overall, readily available affective impression can be easier and more efficient than weighing the pros and cons of various reasons or retrieving relevant examples from memory, especially when the required judgment or decision is complex or mental resources are limited (p. S36),

It would also explain, perhaps, why vaccine endorsement in the US is so high in the first place. Further tests on both the present data and from new studies should look into this. 
However plausible and logical these explanations might be, until more tests can be performed, intuitive cognitive style's directional relation to vaccine endorsement remains veiled in theoretical and statistical questions. What are the details of interaction effects between 'faith in intuition' and conspiracist beliefs upon vaccine endorsement? Is it an artifact in the present sample's data? How should researchers understand the influence of an overall preference toward use of intuition upon personality traits that are themselves founded in intuitions? Such questions must be approached in future research. Until then, present results cannot be taken to support the hypothesis, let alone suggest the plausibility of claims in the literature that an association exists between vaccine hesitancy and the use of heuristics (it does not refute them, either).

\section{Implications: Cognitive style, vaccine endorsement, and affectively held scientific beliefs}

Conclusively, however, results do suggest that analytic thinking predicts vaccine endorsement in the US (see Figure 3), both singularly and holding constant demographic, political, and related psychological variables. This finding is not surprising: it is certainly in line with research linking a willingness and ability to think analytically with higher likelihood of acceptance of mainstream scientific ideas, such as the theory of evolution (see Gervais, 2015), while linking low willingness/ability to think analytically with belief in the paranormal, religious, and other nonscientific beliefs (Browne et al., 2014; Pennycook et al., 2012). That is, given the scientific/biomedical foundations of biomedicine's claims to efficacy, we would expect, looking at the constructs, that acceptance of vaccines would correlate with other traits known to correlate with scientifically founded beliefs.

However, this is certainly not a satisfactory explanation, as it simply equates like-withlike at the level of the concept/stereotype. To look deeper: studies on science learning and developmental psychology have long noted that complex scientific concepts and processes (e.g., 
evolution; gravity) are immensely difficult to grasp even on the rudimentary level, especially compared to everyday concepts (e.g., animal, artifact, natural object), which even very young children seem to grasp with minimal explanation (Chi, Slotta, \& de Leeuw, 1994; Gelman \& Wellman, 1991). Unlike these 'natural' mental concepts, comprehension of complex scientific concepts (e.g., the animal's cellular complexity) is contingent upon sustained effort on the part of the student, and on their ability to override natural intuitions about the world (Chi et al., 1994; Sweller, 1994). All this might imply that the greater one's capacity is to expend analytic mental effort, the more one is likely to grapple with complex scientific concepts. Such gains in familiarity and fluency in complex scientific concepts might, in the long run (at least) nudge one's preferences toward concurrence with the mainstream model of science and medicine, just as it might (at best) engender real expert understanding of scientific topics.

The positive relationship between analytic thinking and vaccine endorsement observed here does not imply that higher capacity to think analytically necessarily leads to greater objective understandings of medicine, immunology, and epidemiology (scientific concepts underlying public vaccination programs). Rather, it implies that for those who's analytic thinking style has led to greater familiarity and fluency with the scientific/medical paradigm, 'truths' inferred about reality are all the more likely to concur with the recommendations of science and medicine (e.g., truths like 'vaccines are a good thing'). Conversely, one with a less analytic cognitive style might be less familiar with such ideas, as they are less likely to spend time in scientific situations, and therefore might be less likely to come to science-based conclusions.

The data seem to bear these conclusions out in an interesting way: in all cognitive style score subgroups (high, medium, and low cognitive style rankings), negatively and positively valenced vaccine endorsement items associated strongly and negatively $(r<-.50)$, indicating 
strongly-affectively (rather than intellectually)-driven decision making regarding vaccines in the sample, even among those with high scores on analytic cognitive style. Among the low, medium, and high 'need for cognition' score subgroups, strength of association increased subsequently per increase in group-rank, suggesting that people with a more analytical cognitive style actually tended to orient toward vaccines more affectively than those with a less analytic cognitive style (although these differences were small, despite being statistically significant ${ }^{5}$ ). This suggests that having a high score on analytic thinking might actually be associated with relatively higher use of affect in coming to vaccine decisions than is the case for a relatively lower score, although again associations for both these score subgroups are strong, as they are in all score subgroups ${ }^{6}$.

This too might suggest that despite analytical cognitive style being associated with higher levels of vaccine endorsement, higher levels of analytic thinking are not necessarily associated with higher likelihood of arriving at vaccine decisions through analytic reasoning. Indeed, results here might suggest that higher vaccine endorsement is more related to a scientific cultural worldview that is influenced by scientific consensuses rather than evaluations of individual data,

5 For instance, 'low' ' "need for cognition'; score subgroup: $r=-.759, p<.001$; 'high' 'need for cognition' score-group: $r=-.931, p<.001$. A one-tailed test after a Fisher's $r$-to- $z$ transformation shows this difference is significant, $z=6.37, p<.001$, while both coefficients are strong. ${ }^{6}$ As for intuitive thinking, while it is interesting that the lowest score sub-group showed the strongest association, and thus the highest level of affective reasoning $(r=-.919, p<001)$, all three associations there were strong and variation was minimal (see Results section), indicating high levels of affective decision making in all three score subgroups, rather than higher scores on 'faith in intuition' being associated with a more affective orientation, as would be expected in light of the hypothesis. 
and which can be intensified through specialized learning (i.e., science education), especially when one possesses the mental capacity ('need for cognition') to at least partially accept, if not understand, the complex principles and concepts that underlie such conclusions.

\section{The role of science education and cultures}

The role of culture and society is crucial in this conjecture. Indeed, the norms of a person's home, society, and culture can influence whether they focus on science at all, no matter what their psychological dispositions. How people come to believe, perceive, and act as they do, at least in ethnographic and psychological literature, is often explained as being derived from the 'truths' that are talked about as real among their groups in day-to-day life (Boyer, 1990;

Luhrmann, 1991; Sobo, 2015; see Zittoun \& Gillespie, 2015). Whatever mechanisms are actually at work in the transmission of cultural norms ${ }^{7}$, they undoubtedly represent base-influences on individual beliefs (Zittoun \& Gillespie, 2015), be these beliefs about the existence of god/spirits; right versus wrong, or the efficacy and validity of medical science. Indeed, social norms are continually implicated in the literature as the major determinant of vaccine attitudes and decisions (Browne et al., 2015; Sobo, Huhn, Sannwald, \& Thurman, 2016; Sobo, 2015). The high level of vaccine endorsement observed in the present sample (which, crucially, is present despite the fact that epidemiological and biomedical concepts are incredibly hard to grasp) might suggest that individuals in mainstream culture internalize the medical 'truths' they encounter just as they do other social norms, and probably employ them without much conscious inspection, irrespective of whether they have a more intuitive or analytic personality. Obviously, the same would be true of groups where non-mainstream beliefs about health and medicine dominate.

\footnotetext{
${ }^{7}$ See Boyer, 1990 for a detailed discussion on the plausibility of various proposed mechanisms cultural transmission.
} 


\section{Conclusion}

Widespread consensus on the safety and necessity of childhood vaccination exists in the U.S. (Kahan, 2014). This is the case despite the fact that most people probably do not understand the complex biomedical and epidemiological concepts that underlie public vaccination programs (see Ritvo et al., 2003). Rather, people likely internalize mainstream medical sentiment as they do other cultural norms: intuitively; automatically, and apart from a large amount of conscious inspection (Boyer, 1990). For some people, the capacity to think scientifically (analytic thinking) might lead to possessing a greater volume of declarative knowledge about vaccine science. However, the present thesis suggests that while analytic thinking does predict vaccine endorsement in the population, endorsement remains the result of an affective appraisal even among those with higher levels of analytic thinking, suggesting that greater vaccine endorsement in this case might arise from a feeling of familiarity and fluency toward science in general among individuals with a higher propensity toward analytic thinking. A positive relationship between intuitive thinking and vaccine endorsement observed in regression models holding constant belief in conspiracies seems to confirm this as well, while at the same time contradicting some claims about the relation of intuitive thinking to vaccine hesitancy. However, lack of statistical clarity there means that specific conclusions on this association must be withheld for now.

\section{Future directions}

Results suggest multiple paths for future research. To begin, repeating this study on both national and cross-cultural samples would test the validity of its conclusions. Future studies might seek out high-and-low vaccine endorsers on both the individual and group level, and might employ psychometrics that better facilitate the testing of hypothesis. For instance, similar studies could be conducted comparing parents of Waldorf (Steiner) School students (who are known to 
medical anthropologists to have comparatively low rates of vaccine endorsement [Sobo, 2015]) with parents of students in traditional education. Studies might also compare the parameters estimated here between students of the natural sciences versus those in other programs.

As for methodological directions, future studies might compare high and low level vaccine endorsers' use of the exact heuristics noted in the literature review as associated with vaccine hesitancy — an idea that was discarded in designing the present study in favor of direct self-reports of intuitive thinking. As is well known to researchers, self-reports can be skewed by any number of factors in the respondent, such as their answering in a way they perceive as being socially desirable (Furnham, 1986), and so this is potentially a problem here. If this association of heuristics and vaccine hesitancy were observed, it would suggest an inverse association between intuitive cognitive style and vaccine endorsement does exist despite that opposite relationships were detected here. Longitudinal designs might also provide a level of validity to conclusions not available through survey research, as use and engagement of both intuitive and analytical cognitive systems changes throughout the lifespan (Epstein, 2014), and age positively predicted vaccine endorsement in the present survey $r_{s}=.110, p<.001$. Lastly, use of experimental rather than survey data should be pursued in later work, as regression analysis is increasingly being criticized by scientists for its high likelihood of disconnect between study constructs and population parameters (see Freedman, Collier, \& Sekhon, 2010).

\section{Limitations}

Multiple limitations were accepted in undertaking this study. First, while Mturk is widely used in academia for survey research, more stringent, or at least more varied sampling methods in future studies would likely increase the validity and generalizability of results. Budgetary constraints were also a factor, and prevented administering the original battery of measurements 
compiled for the research. For instance, the 40-item Core Knowledge Confusion measurement (Lindeman et al., 2008), an arguably more subtle measure of intuitive cognitive style, was omitted from the battery due to budgetary constraints on survey length. Finally, sample weighting according to the Census was not possible because the author had not been trained in this complex undertaking at the time of analysis (fortunately, weighting for a single variable, as was done here, is not complex and easily performed with SPSS). As noted earlier, this does not invalidate the sample, but population parameters could be inferred with greater confidence if it had been weighted in a more sophisticated fashion. Finally, certain statistical procedures were not used here due to the author not having the necessary expertise. Such methods might have revealed additional insights. For instance (and as noted), no interactions between independent variables were entered into the regression models here because the author had not been trained in this advanced procedure. 


\section{References}

Aarnio, K., \& Lindeman, M. (2005). Paranormal beliefs, education, and thinking styles. Personality and Individual Differences, 39, 1227-1236. doi:10.1016/j.paid.2005.04.009

Asch, D. A., Baron, J., Hershey, J. C., Kunreuther, H., Meszaros, J., Ritov, I., \& Spranca, M. (1994). Omission bias and pertussis vaccination. Medical Decision Making, 14, 118-123. doi: $10.1177 / 0272989 \times 9401400204$

Baron, J. (2000). Thinking and deciding. Cambridge, MA: Cambridge University Press. doi: 10.1017/CBO9780511840265

Boom, J., \& Cunningham, R. (2014). Understanding and managing vaccine concerns. Switzerland: Springer International Publishing. doi: 10.1007/978-3-319-07563-1

Boyer, P. (1990). Tradition as truth and communication: a cognitive description of traditional discourse. Cambridge, MA: Cambridge University Press. doi: 10.1017/cbo9780511521058

Brandon, D. M., Long, J. H., Loraas, T. M., Mueller-Phillips, J., \& Vansant, B. (2013). Online instrument delivery and participant recruitment services: Emerging opportunities for behavioral accounting research. Behavioral Research in Accounting, 26, 1-23. doi: $10.2308 /$ bria-50651

Brotherton, R., \& French, C. C. (2014). Belief in Conspiracy Theories and Susceptibility to the Conjunction Fallacy. Applied Cognitive Psychology, 28, 238-248. doi:10.1002/acp.2995 
Brotherton, R., French, C. C., \& Pickering, A. D. (2013). Measuring belief in conspiracy theories: the generic conspiracist beliefs scale. Frontiers in Psychology, 4, 279. doi:10.3389/fpsyg.2013.00279

Browne, M., Pennycook, G., Goodwin, B., \& McHenry, M. (2014). Reflective minds and open hearts: Cognitive style and personality predict religiosity and spiritual thinking in a community sample. European Journal of Social Psychology, 44, 736-742. doi: 10.1002/ejsp.2059

Browne, M., Thomson, P., Rockloff, M. J., \& Pennycook, G. (2015). Going against the Herd: Psychological and Cultural Factors Underlying the "Vaccination Confidence Gap." PloS One, 10, e0132562. doi: 10.1371/journal.pone.0132562

Buchtel, E. E., Norenzayan, A., \& others. (2009). Thinking across cultures: Implications for dual processes. In J. St. B. T. Evans \& K. Frankish (Eds.), In two minds: Dual processes and beyond (pp. 217-238). Oxford, England: Oxford University Press. doi:

10.1093/acprof:oso/9780199230167.003.0010

Center for Disease Control and Prevention. (2006). Resurgence of wild poliovirus type 1 transmission and consequences of importation -- 21 countries, 2002-2005. Morbidity and Mortality Weekly Report, 55, 145-50. Retrieved from http://www.ncbi.nlm.nih.gov/pubmed/16484977

Chaiken, S., \& Trope, Y. (1999). Dual-process theories in social psychology. New York, NY, Guilford Press. 
Chi, M., Slotta, J., \& de Leeuw, N. (1994). From things to Processes: Theory of conceptual change for learning science concepts. Learning and Instruction, 4, 27-43. doi:10.1016/0959-4752(94)90017-5

Coady, D. (2006). Conspiracy Theories: The Philosophical Debate. Burlington, VT: Ashgate Publishing Limited.

Cosmides, L., \& Tooby, J. (1996). Are humans good intuitive statisticians after all? Rethinking some conclusions from the literature on judgment under uncertainty. Cognition, 58, 1-73. doi: 10.1016/0010-0277(95)00664-8

Darwin, H., Neave, N., \& Holmes, J. (2011). Belief in conspiracy theories. The role of paranormal belief, paranoid ideation and schizotypy. Personality and Individual Differences, 50, 1289-1293. doi:10.1016/j.paid.2011.02.027

Diethelm, P., \& McKee, M. (2009). Denialism: What is it and how should scientists respond? European Journal of Public Health, 19, 2-4. doi:10.1093/eurpub/ckn139

Dubé, E., Laberge, C., Guay, M., Bramadat, P., Roy, R., \& Bettinger, J. (2013). Vaccine hesitancy: An overview. Human Vaccines and Immunotherapeutics, 9, 1763-1773. doi:10.4161/hv.24657

Epstein, S. (1994). Integration of the cognitive and the psychodynamic unconscious. The American Psychologist, 49, 709-724. doi:10.1037/0003-066X.50.9.799 
Epstein, S. (1998). Cognitive-experiential self-theory. In D. Barone, M. Hersen, and V.B. Van Hasselt (Eds.) Advanced personality (pp. 211-238). New York, NY: Springer Science + Business Media. doi: 10.1002/9781118133880.hop205005

Epstein, S. (2014). Cognitive-experiential theory: An integrative theory of personality. Oxford, England: Oxford University Press.

Epstein, S., Pacini, R., Denes-Raj, V., \& Heier, H. (1996). Individual differences in intuitiveexperiential and analytical-rational thinking styles. Journal of Personality and Social Psychology, 71, 390-405. doi:10.1037/0022-3514.71.2.390

Evans, J. S. B. T. (2008). Dual-processing accounts of reasoning, judgment, and social cognition. Annual Review of Psychology, 59, 255-278. doi:10.1146/annurev.psych.59.103006.093629

Evans, J. S. B. T. (2012). Questions and challenges for the new psychology of reasoning. Thinking \& Reasoning, 18, 5-31. doi: 10.1080/13546783.2011.637674

Evans, J. S. B. T., \& Stanovich, K. E. (2013). Dual-Process Theories of Higher Cognition: Advancing the Debate. Perspectives on Psychological Science, 8, 223-241. doi:10.1177/1745691612460685

Farias, M., Newheiser, A.-K., Kahane, G., \& de Toledo, Z. (2013). Scientific faith: Belief in science increases in the face of stress and existential anxiety. Journal of Experimental Social Psychology, 49, 1210-1213. doi:10.1016/j.jesp.2013.05.008 
Feldman-Savelsberg, P., Ndonko, F. T., \& Schmidt-Ehry, B. (2000). Sterilizing vaccines or the politics of the womb: retrospective study of a rumor in Cameroon. Medical Anthropology Quarterly, 14, 159-179. doi: 10.1525/maq.2000.14.2.159

Fischhoff, B. (1993). Risk perception and communication. In Fischhoff, B. (Ed.), Risk analysis and human behavior (pp. 3-32). New York, NY: Earthscan.

Frederick, S. (2005). Cognitive reflection and decision making. The Journal of Economic Perspectives, 19, 25-42. Retrieved from: http://www.jstor.org/stable/4134953

Freedman, D. A., Collier, D., \& Sekhon, J. S. (2010). Statistical models and causal inference: a dialogue with the social sciences. Cambridge, MA: Cambridge University Press. 10.1111/j.1751-5823.2010.00122_10.x

Furnham, A. (1986). Response bias, social desirability and dissimulation. Personality and Individual Differences, 7, 385-400. doi: 10.1016/0191-8869(86)90014-0

Gadermann, A. M., Guhn, M., \& Zumbo, B. D. (2012). Estimating ordinal reliability for Likerttype and ordinal item response data: A conceptual, empirical, and practical guide. Practical Assessment, Research \& Evaluation, 17, 1-13. Retrieved from: http://pareonline.net/getvn.asp?v=17\&n=3

Gelman, S., \& Wellman, H. (1991). Insides and essences: Early understan non-obvious. Cognition, 38, 213-244. doi: 10.1016/0010-0277(91)90007-q 
Genovese, J. E. C. (2005). Paranormal beliefs, schizotypy, and thinking styles among teachers and future teachers. Personality and Individual Differences, 39, 93-102. doi: 10.1016/j.paid.2004.12.008

Gervais, W. M. (2015). Override the controversy: Analytic thinking predicts endorsement of evolution. Cognition, 142, 312-21. doi:10.1016/j.cognition.2015.05.011

Gervais, W. M., \& Norenzayan, A. (2012). Analytic thinking promotes religious disbelief. Science, 336(6080), 493-496. doi: 10.1126/science.1215647

Gilovich, T. (1991). How we know what isn't so: The fallibility of human reason in everyday life. New York, NY: Free Press.

Gilovich, T., Griffin, D., \& Kahneman, D. (2002). Heuristics and biases: The psychology of intuitive judgment. Cambridge, MA: Cambridge University Press.

Glanz, J. M., Newcomer, S. R., Narwaney, K. J., Hambidge, S. J., Daley, M. F., Wagner, N. M., ... others. (2013). A population-based cohort study of undervaccination in 8 managed care organizations across the United States. JAMA Pediatrics, 167, 274-281. doi: 10.1001/jamapediatrics.2013.502

Gostin, L. O. (2015). Law, ethics, and public health in the vaccination debates: politics of the measles outbreak. JAMA, 313, 1099-100. doi:10.1001/jama.2015.1518

Gupta, V. B. (2010). Communicating With Parents of Children With Autism About Vaccines and Complementary and Alternative Approaches. Journal of Developmental \& Behavioral Pediatrics 31, 343-345. 10.1097/dbp.0b013e3181d6b6e4 
Jacobson, R. M., Swan, A., Adegbenro, A., Ludington, S. L., Wollan, P. C., Poland, G. A., ... others. (2001). Making vaccines more acceptable methods to prevent and minimize pain and other common adverse events associated with vaccines. Vaccine, 19, 2418-2427. doi: $10.1016 / \mathrm{s} 0264-410 x(00) 00466-7$

Jacobson, R. M., Targonski, P. V., \& Poland, G. a. (2007). A taxonomy of reasoning flaws in the anti-vaccine movement. Vaccine, 25, 3146-3152. doi:10.1016/j.vaccine.2007.01.046

James, W. (1890). The principles of psychology. New York, NY: Henry Holt and Company.

Jolley, D., \& Douglas, K. M. (2014). The effects of anti-vaccine conspiracy theories on vaccination intentions. PLOS ONE, 9. doi:10.1371/journal.pone.0089177

Jost, J. T. (2006). The end of the end of ideology. American Psychologist, 61, 651-670. doi: 10.1037/0003-066X.61.7.651

Kahan, D. M. (2014). Vaccine Risk Perceptions and Ad Hoc Risk Communication: An Empirical Assessment. SSRN Electronic Journal. doi:10.2139/ssrn.2386034

Kahneman, D. (2011). Thinking, fast and slow. New York, NY. Farrar, Straus, and Giroux.

Kanai, R., Feilden, T., Firth, C., \& Rees, G. (2011). Political orientations are correlated with brain structure in young adults. Current Biology : $C B, 21,677-80$. doi:10.1016/j.cub.2011.03.017

Kata, A. (2010). A postmodern Pandora's box: anti-vaccination misinformation on the Internet. Vaccine, 28, 1709-16. doi:10.1016/j.vaccine.2009.12.022 
Kata, A. (2012). Anti-vaccine activists, Web 2.0, and the postmodern paradigm - An overview of tactics and tropes used online by the anti-vaccination movement. Vaccine, 30, 3778-3789. doi:10.1016/j.vaccine.2011.11.112

Klein, G. (1999). Sources of power: How people make decisions. Cambridge, MA: MIT press.

Leman, P. (2007). The born conspiracy. New Scientist, 195, 35-37. doi: 10.1016/s02624079(07)61774-6

Leman, P. J., \& Cinnirella, M. (2007). A major event has a major cause: Evidence for the role of heuristics in reasoning about conspiracy theories. Soc. Psychol. Rev, 9, 18-28. Retrieved from: http://pure.rhul.ac.uk/portal/en/publications/a-major-event-has-a-major-causeevidence-for-the-role-of-heuristics-in-reasoning-about-conspiracy-theories(2ad8342b-f5ca4791-b988-ae70fbfdb4b3).html

Lindeman, M., Cederström, S., Simola, P., Simula, A., Ollikainen, S., \& Riekki, T. (2008). Sentences with core knowledge violations increase the size of N400 among paranormal believers. Cortex, 44, 1307-15. doi:10.1016/j.cortex.2007.07.010

Lobato, E., Mendoza, J., Sims, V., \& Chin, M. (2014). Examining the Relationship Between Conspiracy Theories, Paranormal Beliefs, and Pseudoscience Acceptance Among a University Population. Applied Cognitive Psychology, 28, 617-625. doi: 10.1002/acp.3042

Luhrmann, T. M. (1991). Persuasions of the witch's craft: ritual magic in contemporary England. Cambridge, MA: Harvard University Press. 
Lupton, R., \& Hare, C. (2015). Conservatives are more likely to believe that vaccines cause autism. The Washington Post. Retrieved January 1, 2016, from https://www.washingtonpost.com/blogs/monkey-cage/wp/2015/03/01/conservatives-aremore-likely-to-believe-that-vaccines-cause-autism/

McConnachie, J., \& Tudge, R. (2013). The rough guide to conspiracy theories (3rd ed.). London, England: Rough Guides Ltd.

Meszaros, J. R., Asch, D. A., Baron, J., Hershey, J. C., Kunreuther, H., \& Schwartz-Buzaglo, J. (1996). Cognitive processes and the decisions of some parents to forego pertussis vaccination for their children. Journal of Clinical Epidemiology, 49, 697-703. doi:10.1016/0895-4356(96)00007-8

Nestler, S. (2010). Belief perseverance: The role of accessible content and accessibility experiences. Social Psychology, 41, 35-41. doi:10.1027/1864-9335/a000006

Nickerson, R. S. (1998). Confirmation bias: A ubiquitous phenomenon in many guises. Review of General Psychology, 2, 175. doi: 10.1037/1089-2680.2.2.175

Omer, S. B., Richards, J. L., Ward, M., \& Bednarczyk, R. A. (2012). Vaccination policies and rates of exemption from immunization, 2005--2011. New England Journal of Medicine, 367, 1170-1171. doi: 10.1056/nejmc 1209037

Pacini, R., \& Epstein, S. (1999). The relation of rational and experiential information processing styles to personality, basic beliefs, and the ratio-bias phenomenon. Journal of Personality and Social Psychology, 76, 972-987. doi:10.1037/0022-3514.76.6.972 
Pennycook, G., Cheyne, J. A., Seli, P., Koehler, D. J., \& Fugelsang, J. A. (2012). Analytic cognitive style predicts religious and paranormal belief. Cognition, 123, 335-346. doi: 10.1016/j.cognition.2012.03.003

Poland, C. M., Jacobson, R. M., Opel, D. J., Marcuse, E. K., \& Poland, G. A. (2014). Political, ethical, social, and psychological aspects of vaccinology. In Milligan, G \& Barrett, A (Eds.), Vaccinology: An Essential Guide (pp. 341-349). Oxford, England: John Wiley \& Sons, Ltd. doi: 10.1002/9781118638033.ch20

Poland, C. M., \& Poland, G. A. (2011). Vaccine education spectrum disorder: the importance of incorporating psychological and cognitive models into vaccine education. Vaccine, 29, 6145-61488. doi:10.1016/j.vaccine.2011.07.131

Poland, G. A., \& Jacobson, R. M. (2012). The re-emergence of measles in developed countries: time to develop the next-generation measles vaccines? Vaccine, 30, 103-4. doi:10.1016/j.vaccine.2011.11.085

Poland, G. A., Jacobson, R. M., \& Ovsyannikova, I. G. (2009). Trends affecting the future of vaccine development and delivery: The role of demographics, regulatory science, the antivaccine movement, and vaccinomics. Vaccine, 27, 3240-3244.

doi:10.1016/j.vaccine.2009.01.069

Ritvo, P., Irvine, J., Klar, N., Wilson, K., Brown, L., Bremner, K. E., ... Krahn, M. D. (2003). A Canadian national survey of attitudes and knowledge regarding preventive vaccines. Journal of Immune Based Therapies and Vaccines, 1, 1. doi: 10.1186/1476-8518-1-3 
Rubin, A. (2012). Statistics for evidence-based practice and evaluation. Belmont, CA: Brooks/Cole.

Salmon, D. a, Moulton, L. H., Omer, S. B., DeHart, M. P., Stokley, S., \& Halsey, N. a. (2005). Factors associated with refusal of childhood vaccines among parents of school-aged children: a case-control study. Archives of Pediatrics \& Adolescent Medicine, 159, 470476. doi:10.1001/archpedi.159.5.470

Sladek, R. M., Bond, M. J., \& Phillips, P. A. (2010). Age and gender differences in preferences for rational and experiential thinking. Personality and Individual Differences, 49, 907-911. doi: $10.1016 /$ j.paid.2010.07.028

Slovic, P., Peters, E., Finucane, M. L., \& MacGregor, D. G. (2005). Affect, risk, and decision making. Health Psychology, 24, S35-S40. doi: 10.1037/0278-6133.24.4.S35

Sobo, E. J. (2015). Social cultivation of vaccine refusal and delay among Waldorf (Steiner) school parents. Medical Anthropology Quarterly, 29, 381-399. doi: 10.1111/maq.12214

Sobo, E. J., Huhn, A., Sannwald, A., \& Thurman, L. (2016). Information Curation Among Vaccine Cautious Parents: Web 2.0, Pinterest Thinking, and Pediatric Vaccination Choice. Medical Anthropology. doi: 10.1080/01459740.2016.1145219

Stanovich, K. E., \& West, R. F. (1998). Individual differences in rational thought. Journal of Experimental Psychology: General, 127, 161-188. doi:10.1037/0096-3445.127.2.161

Subbotsky, E. (2014). The belief in magic in the age of science. SAGE Open, 4, 1-17. doi: $10.1177 / 2158244014521433$ 
Swami, V., Voracek, M., Stieger, S., Tran, U. S., \& Furnham, A. (2014). Analytic thinking reduces belief in conspiracy theories. Cognition, 133, 572-585. doi:10.1016/j.cognition.2014.08.006

Sweller, J. (1994). Cognitive load theory, learning difficulty, and instructional design. Learning and Instruction, 4, 295-312. doi: 10.1016/0959-4752(94)90003-5

Tversky, A., \& Kahneman, D. (1973). Availability: A heuristic for judging frequency and probability. Cognitive Psychology, 5, 207-232. doi: 10.1037/e301722005-001

Tversky, A., \& Kahneman, D. (1974). Judgment under uncertainty: Heuristics and biases. Science, 185, 1124-1131. doi: 10.1017/cbo9780511809477.002

Tversky, A., \& Kahneman, D. (1983). Extensional versus intuitive reasoning: The conjunction fallacy in probability judgment. Psychological Review, 90, 293. doi: 10.1016/b978-1-4832$1446-7.50038-8$

West, R. F., Toplak, M. E., \& Stanovich, K. E. (2008). Heuristics and biases as measures of critical thinking: Associations with cognitive ability and thinking dispositions. Journal of Educational Psychology, 100, 930-941. doi:10.1037/a0012842

WHO. (2014). Vaccine-preventable diseases: monitoring system 2014 global summary. Retrieved from http://apps.who.int/immunization_monitoring/globalsummary/countries?countrycriteria[cou ntry][] $=$ USA\&commit $=\mathrm{OK}$ 
Wilson, R., Larson, H., Chiu, J., \& Schulz, W. (2015). HPV Vaccination in Japan. Washington, DC: Center for Strategic \& International Studies. Retrieved from: http://csis.org/files/publication/150422_Wilson_HPVVaccination2_Web.pdf

Zittoun, T., \& Gillespie, A. (2015). Internalization: How culture becomes mind. Culture \& Psychology, 21, 477-491. doi: 10.1177/1354067x15615809 
Appendix A: Tables

Table 1 Education-Level Group Proportions for
Census and Sample Data

\begin{tabular}{|c|c|c|}
\hline Education Level & $\%$ Census $18+$ & \% Survey \\
\hline None & 0.4 & 0 \\
\hline $1 \mathrm{st}-4$ th grade & 0.7 & 0 \\
\hline 5th - 6th grade & 1.4 & 0 \\
\hline 7th - 8th grade & 1.8 & 0.4 \\
\hline 9th grade & 1.6 & 0 \\
\hline 10th grade & 2.0 & 0 \\
\hline 11th grade & 4.4 & 0.5 \\
\hline $\begin{array}{l}\text { High school } \\
\text { graduate }\end{array}$ & 29.6 & 12.5 \\
\hline $\begin{array}{l}\text { Some college no } \\
\text { degree }\end{array}$ & 19.4 & 25.5 \\
\hline $\begin{array}{l}\text { Associate's } \\
\text { degree, } \\
\text { occupational }\end{array}$ & 4.1 & 3.1 \\
\hline $\begin{array}{l}\text { Associate's } \\
\text { degree, } \\
\text { academic }\end{array}$ & 5.3 & 5.4 \\
\hline $\begin{array}{l}\text { Bachelor's } \\
\text { degree }\end{array}$ & 18.9 & 39.9 \\
\hline Master's degree & 7.5 & 10.0 \\
\hline $\begin{array}{l}\text { Professional } \\
\text { degree }\end{array}$ & 1.3 & 0.8 \\
\hline Doctoral degree & 1.6 & 1.8 \\
\hline
\end{tabular}


Appendix A: Tables

Table 2

Age-Range Group Proportions for Census and Sample Data

\begin{tabular}{lcc}
\hline Age Range & \% Census $18+$ & \% Survey \\
\hline 18 to 24 years & 12.6 & 12.6 \\
25 to 29 years & 9.0 & 20.2 \\
30 to 34 years & 8.8 & 18.5 \\
35 to 39 years & 8.1 & 15.4 \\
40 to 44 years & 8.5 & 10.1 \\
45 to 49 years & 8.6 & 4.9 \\
50 to 54 years & 9.3 & 7.8 \\
55 to 59 years & 8.8 & 3.8 \\
60 to 64 years & 7.7 & 4.0 \\
65 to 69 years & 6.2 & 1.8 \\
70 to 74 years & 4.5 & 0.8 \\
75 years and over & 7.8 & 0.2 \\
\hline
\end{tabular}

Note. A Chi Square Goodness of Fit test revealed observed proportions in the sample were highly dissimilar to proportions in the 2014 US census, $\left(\chi^{2}(11)=4.298, p=\right.$ $.960)$ 
Appendix A: Tables

Table 3

Age-Range and Parental Status Proportions

\begin{tabular}{lcc|lcc}
\hline Sex & Frequency & $\%$ & Parental Status & Frequency & $\%$ \\
\hline Male & 267 & 49.2 & Parent & 228 & 41.9 \\
Female & 276 & 50.8 & Non-Parent & 315 & 58.1 \\
Total & 543 & 100.0 & Total & 543 & 100.0 \\
\hline
\end{tabular}


Appendix A: Tables

Table 4

Tests for Independence Between Study Variables and Parental Status

\begin{tabular}{llccc}
\hline Measure & & $\chi^{2}$ & $d f$ & $p$ \\
\hline Psychological & NFC & 22.041 & 20 & .338 \\
& FI & 31.579 & 18 & .025 \\
Conspiracy Theories & CONS & 54.055 & 60 & .692 \\
Political & PO & 17.383 & 4 & .002 \\
Demographic & AGE & 80.155 & 11 & $<.001$ \\
& EDUC & 18.072 & 9 & .034 \\
& SEX & 19.878 & 1 & $<.001$ \\
\hline
\end{tabular}

Note. NFC = need for cognition; FI = faith in intuition; CONS = conspiracist beliefs; $\mathrm{PO}=$ political orientation ( $1=$ very liberal, $5=$ very conservative); EDUC = level of education;; SEX $=\operatorname{sex}(0=$ male, $1=$ female $)$. 
Appendix A: Tables

Table 5

Tests for Independence Between Study Variables and Sex

\begin{tabular}{llccc}
\hline Measure & & $\chi^{2}$ & $d f$ & $p$ \\
\hline Psychological & NFC & 14.510 & 20 & .804 \\
& FI & 40.827 & 18 & .002 \\
Conspiracy Theories & CONS & 61.120 & 60 & .435 \\
Political & PO & 5.334 & 4 & .255 \\
Demographic & AGE & 10.759 & 11 & .464 \\
& EDUC & 3.113 & 9 & .960 \\
& PAR & 19.878 & 1 & $<.001$ \\
\hline
\end{tabular}

Note. NFC = need for cognition; FI = faith in intuition; CONS = conspiracist beliefs; $\mathrm{PO}=$ political orientation ( $1=$ very liberal, $5=$ very conservative); EDUC $=$ level of education; $\mathrm{PAR}=$ parental status $(0=$ non-parent, $1=$ parent). 
Appendix A: Tables

Table 6

Intercorrelations for Ordinal Measures

\begin{tabular}{|c|c|c|c|c|c|c|c|}
\hline Measures & 1 & 2 & 3 & 4 & 5 & 6 & 7 \\
\hline 1. NFC & - & -0.001 & $.154^{\star *}$ & -0.080 & 0.012 & $.109^{\star}$ & $-.135^{\star \star}$ \\
\hline 2. $\mathrm{FI}$ & -0.001 & - & -0.026 & $.205^{\star \star}$ & 0.004 & $-.200^{* *}$ & 0.079 \\
\hline 3. VE & $.154^{\star \star}$ & -0.026 & - & $-.486^{\star *}$ & -0.040 & $.110^{\star *}$ & $-.307^{\star *}$ \\
\hline 4. CONS & -0.080 & $.205^{\star \star}$ & $-.486^{\star *}$ & - & -0.007 & $-.269^{\star *}$ & 0.078 \\
\hline 5. AGE & 0.012 & 0.004 & -0.040 & -0.007 & - & $.092^{*}$ & 0.082 \\
\hline 6. EDU & $.109^{*}$ & $-.200^{\star *}$ & $.110^{* *}$ & $-.269^{\star *}$ & $.092^{*}$ & - & -0.044 \\
\hline 7. $\mathrm{PO}$ & $-.135^{\star \star}$ & 0.079 & $-.307^{\star \star}$ & 0.078 & 0.082 & -0.044 & - \\
\hline
\end{tabular}

Note. $\mathrm{NFC}=$ need for cognition; $\mathrm{FI}=$ faith in intuition; $\mathrm{VE}=$ vaccine orientation; CONS = conspiracist beliefs; $\mathrm{AGE}=$ age; $\mathrm{EDUC}=$ level of education; $\mathrm{PO}=$ political orientation $(1=$ very liberal, $5=$ very conservative).

$* p<.05$

${ }^{* *} p<.001$ 
Appendix A: Tables

\section{Table 7}

Ordinal Regression Models Predicting Vaccine Endorsement

\begin{tabular}{|c|c|c|c|c|c|c|c|c|c|c|c|c|c|}
\hline & & & & & & & Model & & & & & & \\
\hline & & 1 & & 2 & & 3 & & 4 & & 5 & & 6 & \\
\hline Measures & & OR & $95 \% \mathrm{Cl}$ & OR & $95 \% \mathrm{Cl}$ & OR & $95 \% \mathrm{Cl}$ & OR & $95 \% \mathrm{Cl}$ & OR & $95 \% \mathrm{Cl}$ & OR & $95 \% \mathrm{Cl}$ \\
\hline \multirow[t]{2}{*}{ Psychological } & NFC & 1.473 & $1.218,1.782$ & 1.475 & $1.220,1.784$ & 1.401 & $1.148,1.709$ & & & 1.365 & $1.124,1.658$ & 1.336 & $1.089,1.640$ \\
\hline & $\mathrm{FI}$ & & & 0.945 & $0.795,1.146$ & 1.226 & $1.007,1.492$ & & & 1.070 & $0.879,1.301$ & 1.331 & $1.076,1.646$ \\
\hline $\begin{array}{l}\text { Conspiracy } \\
\text { Theories }\end{array}$ & CONS & & & & & 0.931 & $0.919,0.944$ & & & & & 0.928 & $0.914,0.941$ \\
\hline Political & $\mathrm{PO}$ & & & & & & & 0.625 & $0.540,0.724$ & 0.637 & $0.549,0.739$ & 0.610 & $0.522,0.713$ \\
\hline \multirow[t]{4}{*}{ Demographic } & AGE & & & & & & & 0.973 & $0.908,1.042$ & 0.972 & $0.907,1.042$ & 0.977 & $0.908,1.051$ \\
\hline & EDUC & & & & & & & 1.096 & $1.008,1.192$ & 1.090 & $1.000,1.187$ & 0.978 & $0.892,1.074$ \\
\hline & PAR & & & & & & & 1.090 & $0.768,1.545$ & 1.135 & $0.798,1.616$ & 1.209 & $0.834,1.752$ \\
\hline & SEX & & & & & & & 1.175 & $0.846,1.632$ & 1.161 & $0.830,1.625$ & 1.233 & $0.865,1.757$ \\
\hline
\end{tabular}

Note. $\mathrm{OR}=$ odds ratio; $\mathrm{Cl}=$ confidence interval; $\mathrm{NFC}=$ need for cognition; $\mathrm{FI}=$ faith in intuition; $\mathrm{CONS}=$ conspiracist beliefs; $\mathrm{PO}=$ political orientation $(1=$ very liberal, $5=$ very conservative); EDUC $=$ level of education; PAR $=$ parental status $(0=$ non-parent, $1=$ parent $) ; \operatorname{SEX}=\operatorname{sex}(0=\operatorname{male}, 1=$ female $)$. $p$-values $<.05$ shown in bold. 
Appendix B: Figures

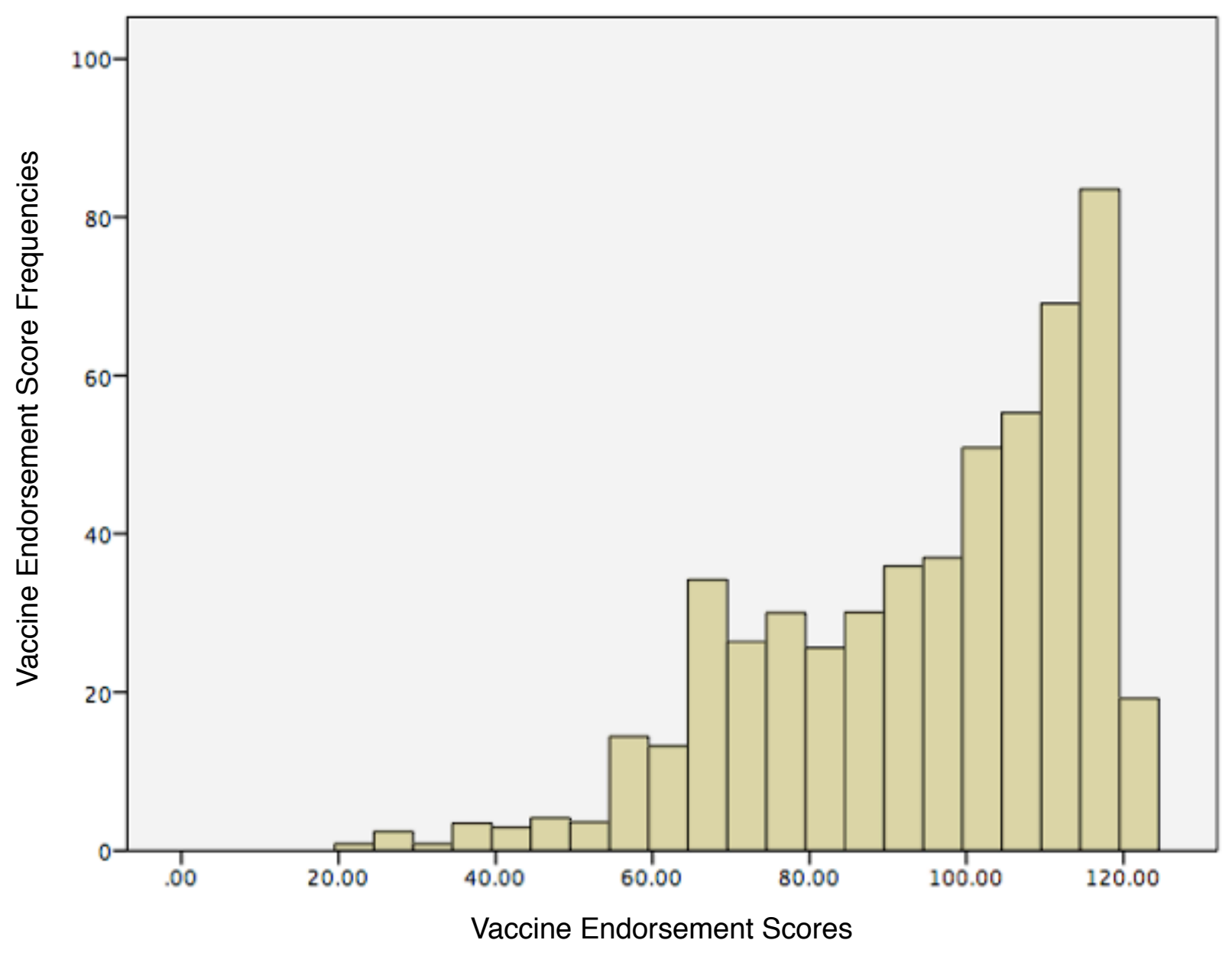

Figure 1. Frequency distributions of Vaccine Endorsement scores. 
Appendix B: Figures

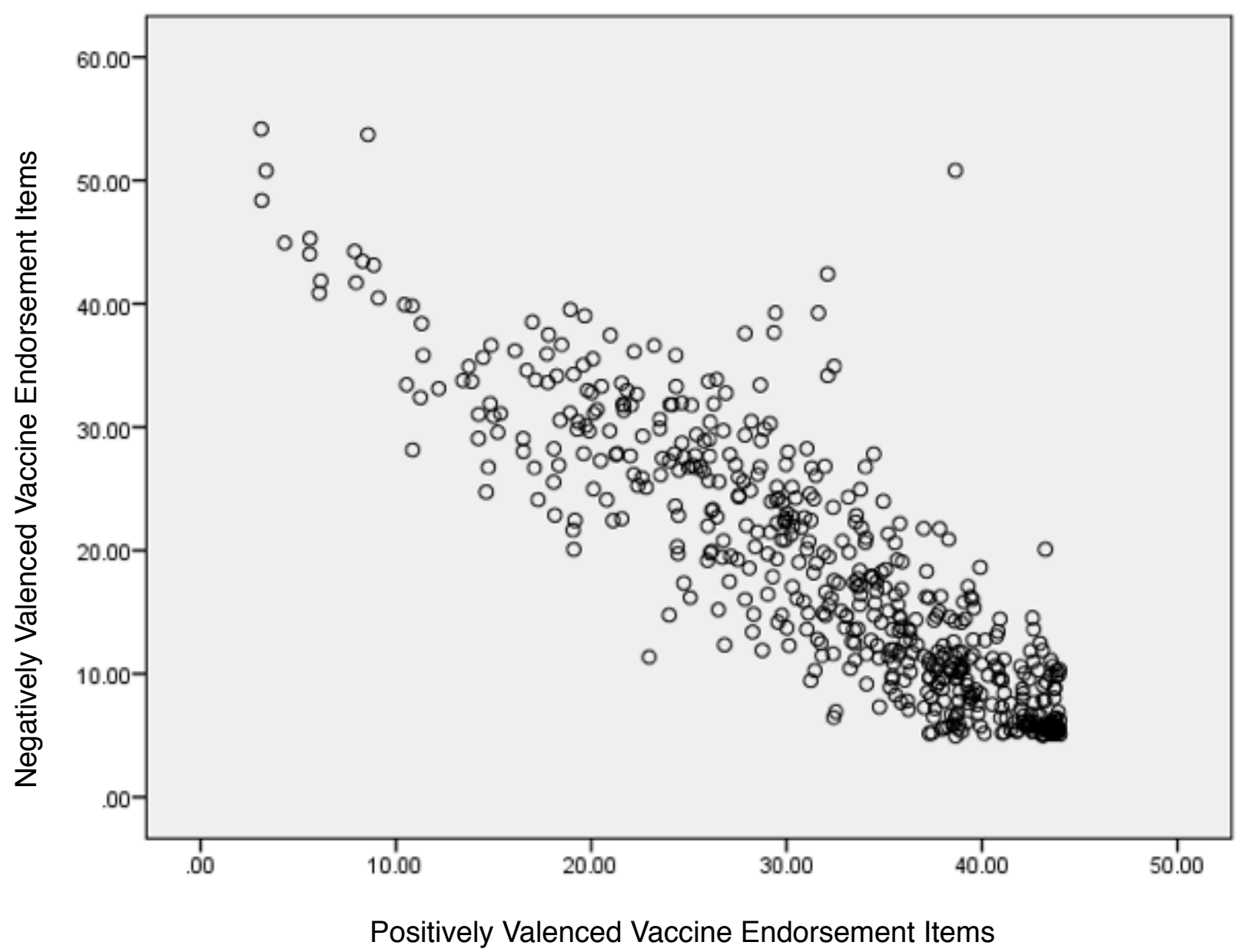

Figure 2. Plotting summed negatively and positively valenced vaccine endorsement items $(r=.872, p<.001)$ 
Appendix B: Figures

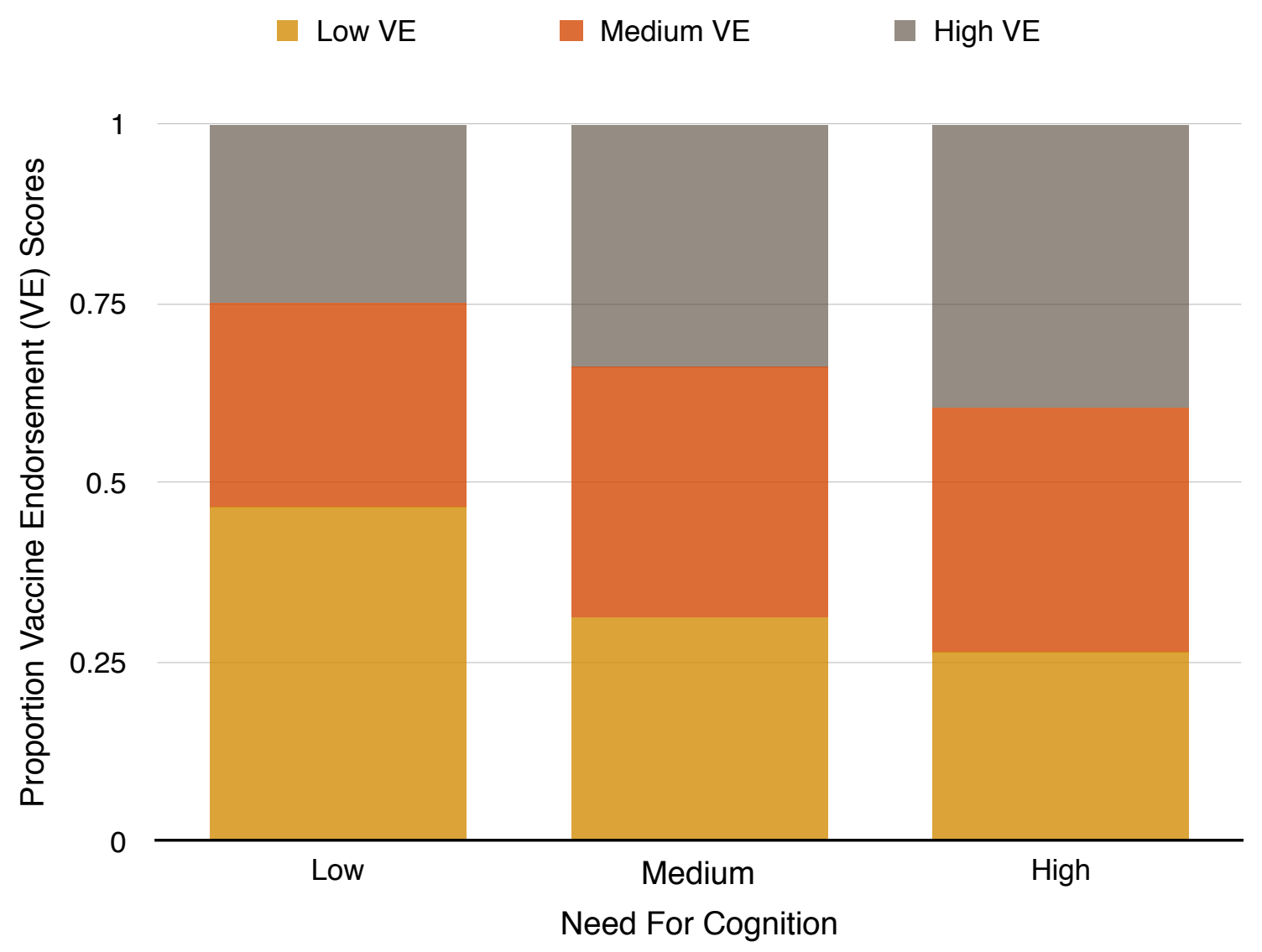

Figure 3. Analytic thinking predicts vaccine endorsement 